\title{
Primer registro de albinismo en el bagre boca chica Ariopsis felis (Siluriformes: Ariidae) del sureste del Golfo de México
}

\section{First record of albinism in hardhead sea catfish Ariopsis felis (Siluriformes: Ariidae) from southeastern Gulf of Mexico}

\author{
Luis A. Guerra-Jiménez ${ }^{1}$ y Raúl E. Lara-Mendoza*2
}

\section{RESUMEN}

Se reporta por primera vez la presencia de un organismo albino de Ariopsis felis capturado por la flota palangrera artesanal de Tabasco a $68.6 \mathrm{~km}$ de la costa y $49 \mathrm{~m}$ de profundidad, el ejemplar correspondió a una hembra sexualmente madura con longitud furcal de $31.5 \mathrm{~cm}$ y peso total de 406.7 g. El presente estudio aumenta los registros de especies albinas en el sureste del Golfo de México.

Palabras clave: Leucismo, alteración genética, bagre, pesquería artesanal, Tabasco.

\section{ABSTRACT}

The presence of an albino Ariopsis felis specimen is recorded for the first time. The specimen was captured by an artisanal longline fleet from Tabasco $68.6 \mathrm{~km}$ off the coast at $49 \mathrm{~m}$ deep. The specimen was a mature female, with $31.5 \mathrm{~cm}$ furcal length and $406.7 \mathrm{~g}$. total weight. This study increases the records of albino species in the southeastern Gulf of Mexico.

Keywords: Leucism, genetic alteration, sea catfish, artisanal fishing, Tabasco.

\section{INTRODUCCIÓN}

El albinismo es una anomalía genética caracterizada por la ausencia total o parcial de la pigmentación en el cuerpo de un organismo, es causada por la alteración de un gen recesivo que afecta la producción de melanina

1 Facultad de Ciencias del Mar, Universidad Autónoma de Sinaloa. Paseo Claussen S/N, Colonia Los Pinos, C. P. 82000

2 Centro Regional de Investigación Pesquera-Ciudad del Carmen, Instituto Nacional de Pesca y Acuacultura. SAGARPA. Av. Héroes del 21 de abril, núm. 26, Col. Playa Norte. Ciudad del Carmen, Campeche. C. P. 24100. rlara. fish@gmail.com* 
(Li et al. 2017). Existen dos tipos de albinismo, en el primero se da una pérdida total de la pigmentación tegumentaria y retinal, y en el segundo una pérdida parcial de la pigmentación en donde solo los ojos o parte del cuerpo presenta la pigmentación original del organismo, a este última también se le conoce como albinismo parcial o leucismo (Lutz, 2001). En peces óseos se han reportado individuos albinos en una amplia variedad de especies marinas y dulceacuícolas, cuyas alteraciones genéticas se han relacionado con factores ambientales, que se podrían originar por diferentes razones como la herencia, la exposición a metales pesados o por selección artificial (Nobile et al. 2016). El comportamiento de los organismos albinos en su hábitat natural es por lo general solitario, lo que los hace vulnerables a depredadores (Hain \& Leatherwood, 1982; Slavík et al. 2015).

El bagre marino boca chica o conocido localmente en Tabasco como curuco, Ariopsis felis (Linnaeus, 1766) es una especie de ambientes tropicales y subtropicales que habita sobre fondos blandos de arena o lodo en aguas salobres y marinas, en conjunto con el bagre bandera (Bagre marinus) representa una de las principales pesquerías en Tabasco, al ser este el estado de mayor producción en el Golfo de México y Mar Caribe mexicano en términos de descarga pesquera (CONAPESCA, 2013). A. felis se encuentra en la cate- goría de menor preocupación de acuerdo al listado de la Unión Internacional para la Conservación de la Naturaleza, sin embargo, no presenta ningún tipo de regulación o medida de protección enfocada a esta especie, por lo que es necesario realizar estudios que aporten información para determinar el estado actual de su población (UICN, 2018). La captura de esta especie se realiza principalmente frente a la Barra de Chiltepec y Frontera, entre los 14-72 $m$ de profundidad, utilizando palangre de fondo con anzuelos circulares, aunque también incide en otras pesquerías con redes de enmalle (DOF, 2012).

El objetivo del presente estudio es reportar por primera vez la presencia de un ejemplar albino del bagre boca chica, A. felis, y aportar información sobre el número de registros de especies que presentan esta anomalía en el sureste del Golfo de México.

\section{MATERIALES Y MÉTODOS}

Durante julio de 2017, se registró un ejemplar $A$. felis, el cual fue capturado por pescadores ribereños de San Pedro, Tabasco (18 32' N, 92 $27^{\circ}$ ' $\mathrm{W})$, utilizando un palangre de fondo con anzuelos circulares (núm. 9/0) y carnada muerta de músculo de bonito (Euthynnus alletteratus), a una distancia de la costa de $68 \mathrm{~km}$ y una profundidad de 49 m (Fig. 1). El ejemplar de A. felis fue determinado utilizando la guía FAO de identificación de especies 
para propósitos pesqueros (Carpenter, 2002), posteriormente, se etiquetó y se llevó al Laboratorio de Investigación Pesquera del Centro Regional de Investigación Pesquera (CRIP), que pertenece al Instituto Nacional de Pesca y Acuacultura con sede en Ciudad del Carmen, Campeche. En el laboratorio, fueron revisadas las características fenotípicas del ejemplar respecto a su coloración; se determinó el sexo mediante la observación directa de las gónadas, se realizaron diversas mediciones morfométricas con un ictiómetro $( \pm 0.1 \mathrm{~cm})$ y se obtuvo el peso total con una balanza digital $( \pm 0.01 \mathrm{~g})$.

\section{RESULTADOS Y DISCUSIÓN}

Se recolectó un ejemplar hembra en condición sexual madura, con longitud furcal (LF) de $31.5 \mathrm{~cm}$ y peso total

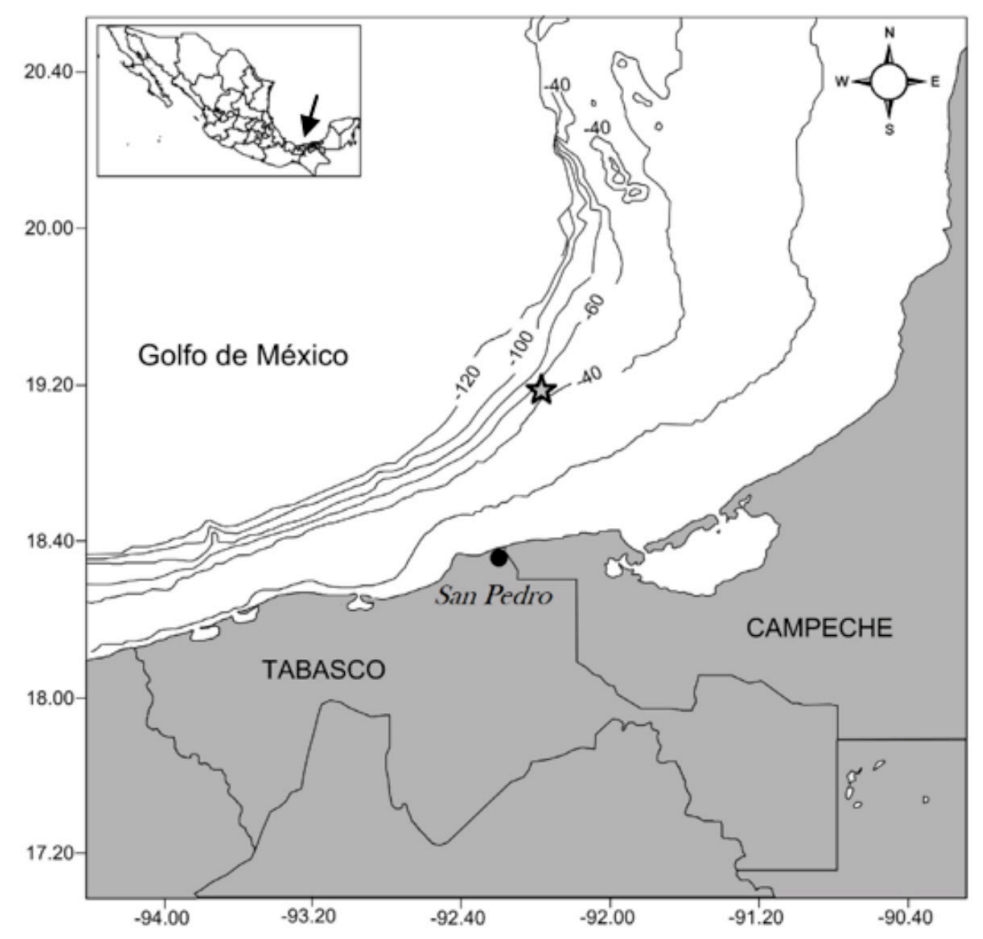

Fig. 1. Localización del sitio de captura (estrella gris) y localidad de desembarque (círculo negro) del ejemplar albino de Ariopsis felis en el sureste del Golfo de México. Los números negativos representan las isobatas en metros

Fig. 1. Catch site (grey star) and fishery landing (black circle) locations of the albino Ariopsis felis specimen in the southeastern Gulf of Mexico. Negative numbers represent the isobates in meters 
(PT) de $406.7 \mathrm{~g}$, otras medidas morfométricas son reportadas en el Cuadro 1. Las características particulares de este organismo fueron su coloración blanca en casi la totalidad de su cuerpo, excepto la segunda aleta dorsal, la cual mostró una coloración más oscura y sus ojos con características similares a la de un organismo normal (Fig. 2), con una coloración marrón azulado en el dorso, que se atenúa a blancuzco en la parte ventral, las aletas pélvicas son pálidas y la segunda dorsal negruzca.

Cuadro 1. Medidas morfométricas del ejemplar albino de Ariopsis felis

Table 1. Morphometric measurements of the albino Ariopsis felis specimen

\begin{tabular}{ll}
\hline Medida & cm \\
\hline Longitud furcal & 35.1 \\
Longitud estándar & 31.5 \\
Altura máxima & 5.6 \\
Contorno máximo & 17.5 \\
Longitud del ojo & 1.3 \\
Longitud de la cabeza & 8.7 \\
Longitud base de la 1. ${ }^{\text {a dorsal }}$ & 3.7 \\
Longitud base de la 2. ${ }^{\text {a dorsal }}$ & 2.1 \\
Longitud base de la aleta pélvica & 1.7 \\
Longitud base aleta pectoral & 1.6 \\
Longitud base aleta anal & 4.6 \\
Longitud primer par de barbillones & 4.5 \\
Longitud segundo par de barbillones & 4.1 \\
Longitud tercer par de barbillones & 2.1 \\
Longitud hocico & 3.1 \\
\hline
\end{tabular}

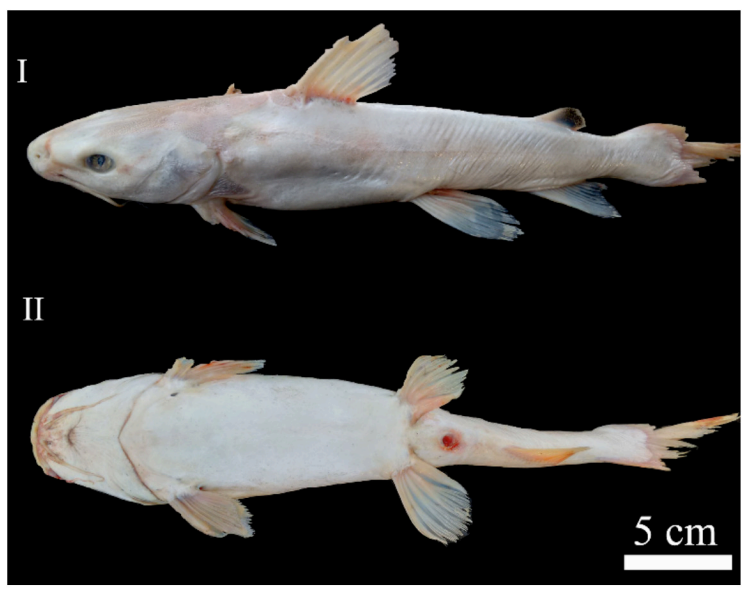

Fig. 2. Vista lateral (I) y ventral (II) de una hembra madura de Ariopsis felis con albinismo, capturada en el sureste del Golfo de México

Fig. 2. Lateral (I) and ventral (II) view of a mature female Ariopsis felis with albinism, captured in southeastern Gulf of Mexico

Este reporte amplía el número de registros de especies de bagre que presentan estas características para el sureste del Golfo de México y para otras partes del mundo (Cuadro 2). Algunos otros registros para la zona han sido para el bagre bandera, Bagre marinus (Wakida-Kusunoki \& Amador-delÁngel, 2013) y la raya americana o balá, Hypanus americanus (WakidaKusunoki, 2015). La presencia de estos eventos de albinismo en especies que habitan el sureste del Golfo de México en los últimos años, puede deberse a diferentes causas, entre ellas la intensa actividad petrolera que hay en la zona y sus derrames (Ponce- 
Velez et al. 2006, Wakida-Kusunoki \& dentro de sus mismas poblaciones Caballero-Chávez, 2009); la presencia (Wakida-Kusunoki \& Amador-delde contenido de metales pesados en Ángel, 2013; Wakida-Kusunoki, los hábitats de estas especies, o el 2015; Mena-Valenzuela \& Valdiviezoresultado de alteraciones genéticas Rivera, 2016).

Cuadro 2. Reportes de anormalidades en la coloración dentro del orden Siluriformes

Table 2. Records of body coloration abnormalities in the Siluriformes order

\begin{tabular}{|c|c|c|c|c|}
\hline $\begin{array}{l}\text { Tipo de } \\
\text { anormalidad }\end{array}$ & Familia & Especie & Localidad & Referencia \\
\hline Albinismo total & Ictaluridae & Ictalurus punctatus & $\begin{array}{l}\text { Río Chickaho- } \\
\text { miny, EE. UU. }\end{array}$ & Menzel (1944) \\
\hline Albinismo total & Ariidae & Arius jella & India & $\begin{array}{l}\text { Gupta \& Bhowmik } \\
\text { (1958) }\end{array}$ \\
\hline Albinismo total & Ariidae & $\begin{array}{l}\text { Tachysurus dussu- } \\
\text { mieri }\end{array}$ & Bahía Palk, India & $\begin{array}{l}\text { Rajapandian \& } \\
\text { Sundaram (1966) }\end{array}$ \\
\hline Albinismo total & Ictaluridae & Noturus gyrinus & $\begin{array}{l}\text { Río Suwannee, } \\
\text { Georgia }\end{array}$ & $\begin{array}{l}\text { Holder \& Ramsey } \\
\text { (1972) }\end{array}$ \\
\hline Albinismo total & Ariidae & $\begin{array}{l}\text { Tachysurus tenuis- } \\
\text { pinis }\end{array}$ & India & Baragi et al. (1976) \\
\hline Albinismo total & Pimelodidae & Zungaro zungaro & & $\begin{array}{l}\text { Taberner et al. } \\
\text { (1976) }\end{array}$ \\
\hline Albinismo total & Ariidae & Arius caelatus & Malpe, India & $\begin{array}{l}\text { Krishna-Pillai \& } \\
\text { Somvanshi (1979) }\end{array}$ \\
\hline Albinismo total & Ictaluridae & Nocturus flavus & $\begin{array}{l}\text { Río Platte, EE. } \\
\text { UU. }\end{array}$ & $\begin{array}{l}\text { Platania et al. } \\
\text { (1986) }\end{array}$ \\
\hline Albinismo total & Heptateridae & Rhamdella minuta & Sao Paulo, Brasil & $\begin{array}{l}\text { Sazima \& Pombal } \\
\text { (1986) }\end{array}$ \\
\hline $\begin{array}{l}\text { Albinismo total } \\
\text { y parcial en } \\
\text { segundo orga- } \\
\text { nismo }\end{array}$ & Siluridae & Silurus glanis & Austria, Francia & $\begin{array}{l}\text { Dingerkus et al. } \\
\text { (1991) }\end{array}$ \\
\hline Albinismo total & Ictaluridae & Ameiurus catis & $\begin{array}{l}\text { Lago Epson, } \\
\text { Gran Bretaña }\end{array}$ & $\begin{array}{l}\text { Britton \& Davies } \\
\text { (2006) }\end{array}$ \\
\hline Albinismo total & Loricariidae & Schizolecis guntheri & Brasil & $\begin{array}{l}\text { de Brito \& } \\
\text { Caramaschi (2005) }\end{array}$ \\
\hline Albinismo total & Ariidae & $\begin{array}{l}\text { Osteogeneiosus } \\
\text { militaris }\end{array}$ & Mumbai, India & $\begin{array}{l}\text { Chavan et al. } \\
\text { (2008) }\end{array}$ \\
\hline Leucismo & Ariidae & $\begin{array}{l}\text { Genidens barbus y } \\
\text { Genidens planifrons }\end{array}$ & Brasil & Leal et al. (2013) \\
\hline Albinismo total & Ariidae & Genidens barbus & Argentina & $\begin{array}{l}\text { Milessi et al. } \\
(2013)\end{array}$ \\
\hline
\end{tabular}




\begin{tabular}{|c|c|c|c|c|}
\hline $\begin{array}{l}\text { Tipo de } \\
\text { anormalidad }\end{array}$ & Familia & Especie & Localidad & Referencia \\
\hline Albinismo total & Ariidae & Bagre marinus & $\begin{array}{l}\text { Sureste Golfo de } \\
\text { México }\end{array}$ & $\begin{array}{l}\text { Wakida-Kusunoki } \\
\text { \& Amador-del- } \\
\text { Ángel (2013) }\end{array}$ \\
\hline Leucismo & Loricariidae & Rhinelepis aspera & Brasil & $\begin{array}{l}\text { Batista-Nobile et } \\
\text { al. (2016) }\end{array}$ \\
\hline $\begin{array}{l}\text { Leucismo } \\
\text { parcial }\end{array}$ & Astroblepidae & Astroblepus ubidiai & $\begin{array}{l}\text { Lago San Pablo, } \\
\text { Ecuador }\end{array}$ & $\begin{array}{l}\text { Mena-Valenzuela } \\
\text { \& Valdiviezo- } \\
\text { Rivera (2016) }\end{array}$ \\
\hline Albinismo total & Heptapteridae & Imparfinis mirini & Sao Paulo & $\begin{array}{l}\text { Manoel et al. } \\
(2017)\end{array}$ \\
\hline Albinismo total & Ariidae & Sciades parkeri & Brasil & $\begin{array}{l}\text { Andrade et al. } \\
\text { (2017) }\end{array}$ \\
\hline $\begin{array}{l}\text { Leucismo } \\
\text { parcial }\end{array}$ & Ariidae & Ariopsis felis & $\begin{array}{l}\text { Sureste Golfo de } \\
\text { México }\end{array}$ & Presente estudio \\
\hline
\end{tabular}

\section{AGRADECIMIENTOS}

A los pescadores de San Pedro, Tabasco, por la donación del ejemplar examinado para fines científicos. Al personal técnico del CRIP-Ciudad del Carmen que apoyaron durante los muestreos y a los revisores anónimos por sus comentarios. El presente estudio formó parte del Proyecto de Investigación Pesquera de los Recursos de Escama Marina de Tabasco, financiado por el Instituto Nacional de Pesca y Acuacultura (INAPESCA).

\section{BIBLIOGRAFÍA}

Andrade, M. C., Schmid, K., Souza, O. \& T. Giarrizzo (2017). First report of albinism in the Threatened Gillbacker sea catfish Sciades parkeri (Siluriformes, Ariidae). Braz. Arch. Biol. Technol., 60, 1-6. e17160326. http://dx.doi. org/10.1590/1678-4324-2017160326
Baragi, M. V., Yagaral, R. B. \& James, P. S. B. R. (1976). On an albino of the marine cat fish Tachysurus tenuispinis (Day). Matsya 2, 82-83.

Batista-Nobile, A., Freitas-Souza, D., Pontieri de Lima, F., Acosta, A. A. \& Da Silva, R. J. (2016). Partial albinism in Rhinelepis aspera of albinismo in South American freshwater fishes. Rev. Mex. Biodivers., 87(2), 531-534. https://doi. org/10.1016/j.rmb.2016.04.005

Britton, T. R. \& Davies, G. D. (2006). First record of the catfish Ameiurus catus in Great Britain. J. Fish Biol. 69(4), 12361238. https://doi.org/10.1111/j.10958649.2006.01171.x

Carpenter, K. E. (2002). The living marine resources of the western central Atlantic. Roma, Italia. FAO.

Chavan, B. B., Sundaram, S., Sawant, A. D. \& Das, T. (2008). Record of complete albinism in marine catfish Osteogeneiosus militaris (Linnaeus 1758), landed at Mumbai. Mar. Fish. Inform. Serv., 197, 15-17.

CONAPESCA. (2013). Anuario estadístico de acuacultura y pesca 2013. Mazatlán, 
Sinaloa, México: Comisión Nacional de Acuacultura y Pesca. SAGARPA.

de Brito, M. F. G. \& Caramaschi, P. E. (2005). An albino armored catfish Schizolecis gunteri (Siluriformes: Loricariidae) from an Atlantic Forest coastal basin. Neotrop. Ichthyol. 3(1), 123-125. https://doi.org/10.1590/ S1679-62252005000100009

Dingerkus, G., Séret, B. \& Guilbert, E. (1991). The first albino wels, Silurus glanis Linnaeus, 1758, from France, with a review of albinism in catfishes (Teleostei: Siluriformes). Cybium, 15(3), 185-188.

DOF. (2012). Acuerdo mediante el cual se da a conocer la actualización de la Carta Nacional Pesquera (continúa en la Tercera Sección). (Publicado en DOF: 2/12/2010). México. Diario Oficial de la Federación.

Gupta, P. D. \& Bhowmik, R. M. (1958). A case of albinism in Arius jella Day from India. Sci. Cult., 24(6), 283.

Hain, J. H. \& Leatherwood, S. (1982). Two sightings of white pilot whales, Globicephalia melaena and summarized records of anomalously white cetaceans. J. Mammal., 63(2), 338-343. https:// doi.org/10.2307/1380654

Holder, D. R. \& Ramsey, J. S. (1972). A case of albinism in the Tadpole Madtom, Noturus gyrinus. Trans. Am. Fish. Soc. 101(3), 566-567. https://doi.org/10.1577/1548-8659(1972)101\%3C566b:ACOAIT\%3E2.0.CO;2

Krishna-Pillai, S. \& Somvanshi, V. S. (1979). A case of complete albinism in marine cat fish Arius caelatus (Valenciennes). Indian J. Fish., 26(1/2), 240-241.

Leal, E. M., Schulz, H. U., Albornoz, L, P., Manchado, R. \& Ott, H. P. (2013). First record of Partial Albinism in two Catfish Species of Genidens (Siluriformes: Ariidae) in an Estuary of Southern Bra- zil. Braz. Arch. Biol. Technol. 56(2),

237-240. https://doi.org/10.1590/ S1516-89132013000200008

Li, Y., Geng, X., Bao, L., Elaswad, A., Huggins, W. K., Dunhamm, R. \& Liu, Z. (2017). A deletion in the Hermansky-Pudlak syndrome $4\left(H_{p s} 4\right)$ gene appears to be responsible for albinism in channel catfish. Mol. Genet. Genomics, 292, 663-670. https://doi.org/10.1007/ s00438-017-1302-8

Lutz, C. G. (2001). Gene Action I: Qualitative Traits. In C. G. Lutz (Ed.), Practical Genetics for Aquaculture (pp. 4-32). Iowa, EE. UU.: A Blackwell Science Ltd. https://doi. org/10.1002/9780470999837

Manoel, S. P., Ono, E. R. \& Alves, M. I. B. (2017). First report of albinism in the South American catfish Imparfinis mirini (Siluriformes: Heptapteridae). Rev. Mex. Biodivers., 88(2), 471-473. https://doi.org/10.1016/j. rmb.2017.01.030

Mena-Valenzuela, P. \& Valdiviezo-Rivera, J. (2016). Leucismo en Astroblepus ubidiai (Pellegrin 1931) (Silufirmes: Astroblepidae), de la provincia de Imbabura, Ecuador. Biota Colombiana, 17(1), 131-136. https://doi. org/10.21068/c2016v17r01a10

Menzel, R. W. (1944). Albino catfish in Virnigia. Copeia, 2, 124. https://doi.org/10.1016/j.rmb.2017.01.030

Milessi, C. A., Cortés, F. \& Jaureguizar, A. (2013). First report of albinism in the marine catfish Genidens barbus (Lacepède 1803) in Argentine waters. Pan-American J. Aquat. Sci., 8 (2), 139-141.

Nobile, B. A., Freitas-Souza, D., Pontieri de Lima, F., Acosta, A. A. \& Da Silva, R. J. (2016). Partial albinism in Rhinelepis aspera from the upper Paraná Basin, Brazil, with a review of 
albinism in South American freshwater fishes. Rev. Mex. Biodivers., 87(2), 531-534. https://doi.org/10.1016/j. rmb.2016.04.005

Platania, P. S., Cummings, T. R., Kehmeier, K. J. (1986). First verified record of the Stonecat, Nocturus (Ictaluridae), in the South Platte River System, Colorado, with notes on an albinistic specimen. Southwest. Nat., 31(4), 553-555. https://doi.org/10.2307/3671721

Ponce-Vélez, G., Botello, A. V. \& Díaz-González, G. (2006). Organic and inorganic pollutants in marine sediments form northern and southern continental shelf of the Gulf of Mexico. Int. J. Environ. Poll., 26(1/2/3), 295-304. https://doi. org/10.1504/IJEP.2006.009113

Rajapandian, M. E. \& Sundaram, K. S. (1967). A case of complete albinism in the catfish Tachysurus dussumieri (Cuvier and Valenciennes). J. Mar. Biol. Ass. India, 9(1), 194-195.

Sazima, I. \& Pombal Jr., J. (1986). Um albino de Rhamdella minuta, com notas sobre comportamento (Osteichthyes, Pimelodidae). Rev. Bras. Biol., 46(2), 377-381.

Slavík, O., Horhy, P. \& Maciak, M. (2015). Ostracism of an Albino Individual by a group of pigmented Catfish. PLOS ONE 10(5), e0128279. https://doi. org/10.1371/journal.pone.0128279
Taberner, R., Fernández-Santos, J. O. \& Castelli, J. O. (1976). Un manguruyú albino Plaulicea leutkeni (Steindachner, 1876) Eigenmann, 1910. Physys. B., 35(91), 121-123.

UICN. (2018). The IUCN Red List of Threatened Species. Recuperado en marzo 6, 2018, disponible en http:// www.iucnredlist.org

Wakida-Kusunoki, A. T. \& Amador-delÁngel, L. E. (2013). First record of albinismin gafftopsail catfish Bagre marinus (Pisces: Ariidae) from southeast México. Rev. Biol. Mar. Oceanogr., 48(1), 203-206. https://doi.org/10.4067/S071819572013000100019

Wakida-Kusunoki, A. T. \& Caballero-Chávez, V. (2009). Efectos del derrame de hidrocarburos del pozo Kab sobre la pesca ribereña en el litoral de Campeche y Tabasco, México. Cienc. Pesq., 17(1), 65-73.

Wakida-Kusunoki, A. T. (2015). First record of total albinism in southern stingray Dasyatis americana. Rev. Biol. Mar. Oceanogr., 50(1), 135139. https://doi.org/10.4067/S07181957201500010001 